\title{
Drugs as diagnostic tools
}

\section{Dexamethasone and Cushing syndrome}

\author{
Nicole Arseneau, Carlos Muzlera \\ Faculty Reviewer: Stan Van Uum, MD, PhD, FRCPC (Department of Medicine; Division of Clinical Pharmacology; Division of Endo- \\ crinology and Metabolism)
}

\section{MR SIMON}

Mr Simon, age 36, presents to his family physician with concerns about his weight. He has gained $20 \mathrm{~kg}$ in the past 4 months, and has developed purple striae on his abdomen. His body mass index (BMI) is currently 34 , and on physical examination, he is found to have a rounded face, a large dorsal fat pad, and a waist circumference of $112 \mathrm{~cm}$. His blood pressure is elevated at 145/95 $\mathrm{mmHg}$, while all other vitals are normal. He also mentions worsening acne on his face, shoulders, and upper back, as well as mild depression for the past 3 months. His past medical history is significant only for an appendectomy at age 10. He has no family history of diabetes, obesity, or cardiac disease. The only medication he has been on for the past 3 years is a multivitamin supplement. He does not smoke, drinks approximately 5 alcoholic drinks per week, and does not take any illicit drugs.

\section{INITIAL INVESTIGATIONS}

Mr Simon is presenting with the classic fat redistribution pattern indicative of Cushing syndrome, a pattern of signs and symptoms which can occur after chronic exposure to excess glucocorticoids (hypercortisolism). ${ }^{1}$ The most common cause of Cushing syndrome is exogenous glucocorticoid administration, usually due to chronic steroid use for inflammatory, neoplastic, or autoimmune disorders. In these cases, supraphysiologic doses of exogenous steroids will both cause Cushingoid features as well as suppress endogenous corticosteroid production. ${ }^{2-4}$ Thus, when treating these patients, it is necessary to remove the offending drug slowly by tapering doses down over weeks or even months to ensure that no withdrawal occurs, as adrenal hormone insufficiency is deadly in its own right. ${ }^{2,5}$ In Mr Simon's case, exogenous glucocorticoid administration is unlikely as he has no history of taking steroids for any condition, thus his physician must search for other causes of his symptoms. It is possible that Mr Simon's weight gain and hypertension could be a result of a sedentary lifestyle, however the rapidity of the weight gain, other classic Cushingoid features (moon facies, increased dorsal fat pad, purple striae), and androgenic features (worsening acne) still strongly suggest Cushing syndrome., ${ }^{1,3}$ In order to confirm her suspicions, Mr Simon's physician orders a 24 hour urinary free cortisol measurement as well as midnight salivary cortisol tests to confirm hypercortisolism. The physician also orders a fasting blood glucose measurement as well as a lipid panel to investigate for diabetes mellitus and metabolic syndrome. ${ }^{4,6}$

Mr Simon's 24 hour urinary free cortisol level is elevated at 872 $\mathrm{nmol} / \mathrm{d}$ (normal $30-300 \mathrm{nmol} / \mathrm{d}$ ). A repeated measurement is also elevated at $931 \mathrm{nmol} / \mathrm{d}$. His midnight salivary cortisol levels are both also elevated at $11.2 \mathrm{nmol} / \mathrm{L}$ and $10.7 \mathrm{nmol} / \mathrm{L}$ (normal 3.0-4.0 nmo$l / L)$. Both blood glucose and lipids are within normal limits. He is referred to an endocrinologist for diagnosis and treatment of hypercortisolism.

\section{DETERMINING THE SOURCE}

With hypercortisolism confirmed, the endocrinologist's task is to determine the source of the excess cortisol. As discussed previously, exogenous glucocorticoid administration has been excluded based on the patient's history, so endogenous causes must be considered. In a healthy person, cortisol secretion is regulated by the hypothalamus and the pituitary through the hypothalamic-pituitary-adrenal (HPA) axis (Figure). In times of stress, the hypothalamus secretes corticotropin-releasing hormone (CRH) which acts on the pituitary to stimulate adrenocorticotropic hormone (ACTH) secretion. ACTH then acts on the adrenal cortex to stimulate cortisol release. When there is cortisol in the blood, it acts on both the hypothalamus and pituitary to suppress the release of any further $\mathrm{CRH}$ or ACTH, thus acting as negative feedback and inhibiting its own release. ${ }^{6,7}$ However, if any of these regulatory mechanisms fail, a disease state such as Cushing syndrome can result. Endogenous Cushing syndrome can thus be classified into ACTH-dependent ( $80 \%$ of Cushing syndrome cases) or ACTH-independent (20\%) subtypes. In ACTH-dependent disease, the excess amounts of ACTH are released by the pituitary or another tumor, which stimulates the adrenal glands to produce excess cortisol. The most common cause is Cushing's disease, in which a pituitary adenoma secretes the excess ACTH. In ACTH-independent disease, most

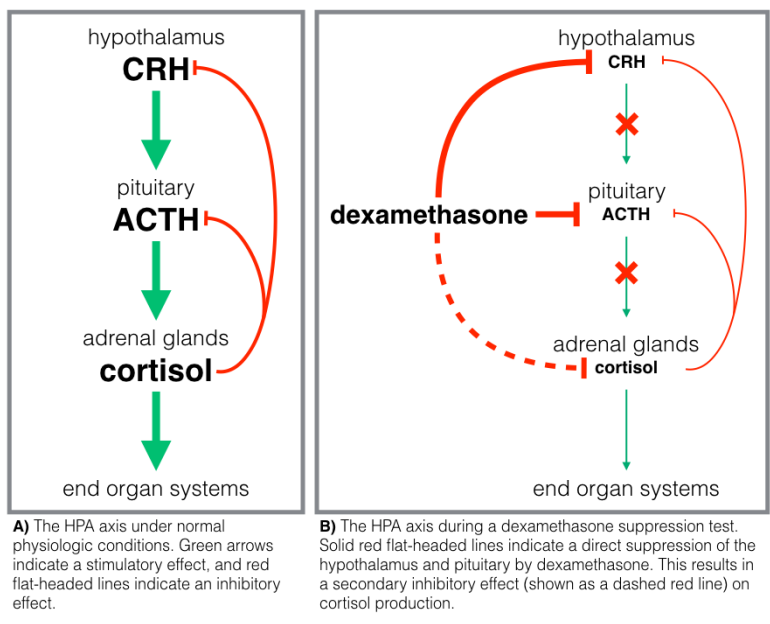

Figure. Hypothalamic-pituitary-adrenal axis. 
commonly due to an adrenal adenoma, one or both adrenal glands functions autonomously to produce excess cortisol regardless of ACTH stimulation. ${ }^{3,4,6}$

In order to test whether these regulatory mechanisms are intact, the endocrinologist performs a low-dose dexamethasone overnight suppression test (LDDST). In this test, $1 \mathrm{mg}$ of dexamethasone, an orally administered corticosteroid medication, is given to the patient at bedtime, and serum cortisol is measured the next morning. ${ }^{1,4,8,9}$ This is an example of a unique subset of tests where medications are used to mimic endogenous compounds to elicit expected results. In a healthy patient, the dexamethasone should act like cortisol to suppress $\mathrm{CRH}$ and $\mathrm{ACTH}$, which should result in reduced cortisol secretion overnight, and thus a serum cortisol level of $<50 \mathrm{nmol} / \mathrm{L}$ the next morning. If the cortisol level is suppressed the next morning, this suggests that the source of endogenous cortisol is ACTH-dependent and nonautonomous. If no suppression occurs, the source is more likely to be autonomous and nonACTH-dependent. ${ }^{1,4,8,9}$ Caution must be used when performing the LDDST, as common medications such as estrogen supplementation (in oral contraceptive pills) can affect total cortisol levels, and drugs such as carbamazepine and rifampin can increase metabolism of dexamethasone itself, such that it insufficiently suppresses cortisol. Use of these medications during the test or in the weeks prior to it could result in an inaccurate test. ${ }^{6,8} \mathrm{~A}$ high-dose dexamethasone test can also be performed in certain clinical scenarios when a lowdose test is inconclusive. ${ }^{9,10}$

In some centres, a direct analysis of serum ACTH is also available, which can help further differentiate ACTH-dependent and -independent sources of cortisol. ${ }^{4}$ For example, if there is a pituitary abnormality resulting in increased ACTH production (which in turn causes increased cortisol secretion), serum ACTH levels would be high (or inappropriately normal for the high cortisol secretion). If there is an adrenal source of cortisol that is autonomous while the pituitary remains normal, high serum levels of cortisol would suppress ACTH production, thus it would be measured low in the serum. To be thorough, Mr Simon's endocrinologist orders both a low-dose dexamethasone test as well as a serum ACTH level.

$M r$ Simon's serum cortisol fails to suppress after a LDDST, indicating that cortisol secretion is no longer regulated by the HPA axis. His serum ACTH level is undetectable. His endocrinologist concludes that the patient's Cushing's syndrome is ACTH-independent, and therefore suspects an adrenal source. Mr Simon is sent for an abdominal computed tomography (CT) scan, which identifies a well defined, noninvasive $4.6 \mathrm{~cm}$ mass in his left adrenal gland, while the right adrenal gland appears atrophic.

\section{MANAGEMENT OF CUSHING SYNDROME}

Mr Simon is diagnosed with an adrenal cortical adenoma. The endocrinologist consults the imaging studies and concludes that, as there is no local invasion or evidence of metastases, the mass is benign. Since the laboratory investigations indicated that the mass is hormonally active and is producing symptoms, $\mathrm{Mr}$ Simon is scheduled for surgical removal of the mass in 6 weeks. If the severity or progression of his symptoms is worrisome, he may be prescribed an inhibitor of glucocorticoid synthesis, such as ketoconazole, to reduce his cortisol production while he waits for surgery. If he is not a surgical candidate, glucocorticoid synthesis inhibitors may be continued as treatment. ${ }^{11,12}$

The best course of action for Mr Simon is to remove the mass in his adrenal gland that is producing cortisol. ${ }^{11,12}$ Meanwhile, his contralateral adrenal gland has become atrophic from lack of stimulation, as the excess cortisol produced by the tumor is suppressing ACTH at the pituitary level. Thus, when the adrenal cortical adenoma is removed, the contralateral adrenal gland will not be able to function adequately until it can recover from atrophy. For this reason, it is necessary to give $\mathrm{Mr}$ Simon oral corticosteroid therapy postoperatively so that he does not experience an adrenal insufficiency crisis, which can be life threatening. Generally, this therapy must continue for weeks to months, based on his recovery, and he must be weaned off the medication slowly to further reduce his risk for adrenal insufficiency. Thus, while surgery is the preferred treatment option for a patient with an adrenal cortical adenoma, medications play an extremely important role in ensuring good outcomes for the patient. Postoperatively, he must be monitored closely to ensure that no other complications of his surgery arise, and that his other concerns (weight gain, acne, depression) resolve with treatment. . $^{3,6,11,12}$

Mr Simon successfully undergoes a unilateral left adrenalectomy. Postoperatively he is given $50 \mathrm{mg}$ of hydrocortisone intravenously trice daily, which is gradually changed to oral administration and tapered to $40 \mathrm{mg}$ daily. He is followed closely, and his steroid medication is further tapered successfully at 6 months with no medications being taken at 9 months. At 1 year after surgery, Mr Simon has a BMI of 25, a waist circumference of $88 \mathrm{~cm}$, blood pressure of 125/85, and reports that his depression has improved greatly.

\section{REFERENCES}

1. Nieman LK. Cushing's syndrome: update on signs, symptoms and biochemical screening. Eur J Endocrinol. 2015;173(4):M33-8.

2. Hopkins RL, Leinung MC. Exogenous Cushing's syndrome and glucocorticoid withdrawal. Endocrinol Metab Clin North Am. 2005;34:37184.

3. Nieman LK, Feelders RA, Kennedy E. Cushing's syndrome: epidemiology and developments in disease management. Clin Epidemiol. 2015:281-93.

4. Nieman LK, Biller BMK, Findling JW, Newell-Price J, Savage MO, Steward PM, et al. The diagnosis of cushing's syndrome : an endocrine society clinical practice guideline. 2008;93(May):1526-40.

5. Fraser LA, Van Uum S. Work-up for Cushing syndrome. CMAJ. 2010;182(6):584-7.

6. Newell-Price J, Bertagna X, Grossman AB, Nieman LK. Cushing's syndrome. Lancet. 2006;367(9522):1605-17.

7. Newell-Price J, Trainer P, Besser M, Grossman A. The diagnosis and differential diagnosis of cushing's syndrome and pseudo-cushing's states. Endocr Rev. 1998;19(5):647-72.

8. Hempen C, Elfering S, Mulder AHL, van den Bergh FAJTM, Maatman RGHJ. Dexamethasone suppression test: development of a method for simultaneous determination of cortisol and dexamethasone in human plasma by liquid chromatography/tandem mass spectrometry. Ann Clin Biochem. 2012;49(Pt 2):170-6

9. Reimondo G, Allasino B, Bovio S, Saba L, Ardito A, Angeli A, et al. Pros and cons of dexamethasone suppression test for screening of subclin- 


\section{Bluewater Health Sarnia \& Petrolia, Ontario}

Bluewater Health is recruiting for the following positions to provide services to the residents of Sarnia-Lambton both in the community and at the hospital.

- Paediatrician

- Hospitalists

- Internists with Endocrinology or Rheumatology interest
- Neurologist

- Psychiatrist

- Emergency Physicians

- Rheumatologist
- Orthopaedic Surgeon

- Dermatologist

- Infectious Disease (Part Time)

Bluewater Health operates 326 beds at hospitals in Petrolia and Sarnia. We are award winning, Accredited with

Exemplary Standing, and have state-of-the-art facilities. We offer an array of specialized acute, complex continuing care, allied health and ambulatory care services including:

- A broad range of diagnostic services, including CT, and MRI.

- Full spectrum of specialists available 24/7 (Neurosurgery and Interventional Cardiology not available)

- Closed Critical Care Unit - District Stroke Centre - Schedule 1 Psychiatric Unit

-Affiliation with Western University's Schulich Medicine Distributed Education.

We are committed to improving the patient experience using engagement, lean, and innovation.

Situated on the shores of Lake Huron at the border to Michigan,

Sarnia offers excellent quality of life, sports, arts and culture, education, state of the art health care, and beautiful natural environment.

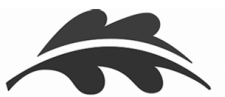

To inquire about these positions, please contact:

Dr. Mike Haddad, Interim Chief of Professional Staff

Bluewater Health

Email: Medical_affairs@bluewaterhealth.ca Tel: 519-464-4400 ext 4534.

BLUEWATER

H E A L T H

Life, health and renewal.

ical Cushing's syndrome in patients with adrenal incidentalomas. J

Endocrinol Invest. 2011;34(1):1-5.

10. Grossman R. Dexamethasone Suppression Test Findings in Subjects With Personality Disorders: Associations With Posttraumatic Stress Disorder and Major Depression. Am J Psychiatry. 2003;160(7):1291-8.

11. Bittner IV JG, Brunt LM. Evaluation and management of adrenal incidentaloma. J Surg Oncol. 2012;106(5):557-64.

12. Nieman LK, Biller BMK, Findling JW, Murad MH, Newell-Price J, Savage MO, et al. Treatment of Cushing's Syndrome: An Endocrine Society Clinical Practice Guideline. J Clin Endocrinol Metab. 2015;100(8):2807-31. 